\title{
Fluorescence Near-Field Microscopy of DNA at Sub-10 nm Resolution
}

\author{
Ziyang Ma, ${ }^{*}$ Jordan M. Gerton, ${ }^{\dagger}$ Lawrence A. Wade, and Stephen R. Quake* \\ Applied Physics and Physics, California Institute of Technology, Pasadena, California 91125, USA
}

(Received 21 October 2005; published 27 December 2006)

\begin{abstract}
We demonstrate apertureless near-field microscopy of single molecules at sub-10 nm resolution. With a novel phase filter, near-field images of single organic fluorophores were obtained with $\sim$ sixfold improvement in the signal-to-noise ratio. The improvement allowed pairs of molecules separated by $\sim 15 \mathrm{~nm}$ to be reliably and repeatedly resolved, thus demonstrating the first true Rayleigh resolution test for near-field images of single molecules. The potential of this technique for biological applications was demonstrated with an experiment that measured the helical rise of $A$-form DNA.
\end{abstract}

PACS numbers: 07.79.Fc, 42.50.Hz, 87.15.-v, 87.64.Xx

For nano- and molecular science and technology, nearfield optical microscopy provides a technique to measure and manipulate structures at subdiffraction limited resolution. The use of a sharp apertureless tip to locally perturb the fields at the sample with apertureless near-field scanning optical microscopy (ANSOM) has allowed spatial resolution at or surpassing $20 \mathrm{~nm}$ using elastic scattering [1,2], Raman scattering [3,4], and fluorescence excitation $[5,6]$. With fluorescence ANSOM, fluorescence of the sample is modified by the proximity of the tip that enhances the excitation field near it, but at the same time induces nonradiative energy transfer (fluorescence quenching) [7]. As a result of the two competitive effects, only single folds of fluorescence enhancement [8-14] or small fractions of fluorescence quenching $[15,16]$ can be measured. Detection of the small high-resolution signal against the classical signal excited by the laser illumination has remained the main concern of fluorescence ANSOM.

Single molecules are widely used as fluorescent tags or reporters in biology [17], sensitive probes in materials and physical chemistry [18], and model single quantum systems for studying light-matter interactions [18]. Near-field optical imaging of single molecules has intrigued scientists since the demonstration by Betzig et al. [19]. Unfortunately, it has been a challenge $[7,16]$ to image fluorescent molecules with ANSOM due to the inherent molecular fluorescence fluctuation [inset of Fig. 1(b)] and the limited number of photons available before photochemical destruction (photobleaching) of the molecule. Only two experiments have achieved resolution at $30-40 \mathrm{~nm}$ by imaging isolated molecules in vacuum or in a matrix [11] or using a nanofabricated metal tip on top of a fiber aperture [20]. More recently, it was demonstrated that properly designed "nanoantennas" can enhance the power of the optical near field by several orders $[21,22]$ or reduce nonradiative energy transfer [23], thus holding promise for imaging single molecules. In this Letter, we demonstrate single-molecule ANSOM imaging at sub-10 $\mathrm{nm}$ resolution using a novel phase filter. For the first time, two molecules separated by less than $15 \mathrm{~nm}$ can be resolved with
ANSOM. We applied this technique to measure the helical rise of $A$-form DNA. The progress we present will accelerate the application of fluorescence ANSOM in the life sciences.

The microscope setup was described previously [6]. Briefly, an atomic force microscope (tapping mode: $\sim 80 \mathrm{kHz}$ ) is combined with an inverted confocal optical microscope, with the silicon tip (FESP, Veeco Instruments) aligned with the laser focal spot [Fig. 1(a)]; the fluorescence photons and the beginning of the tip oscillation cycles were recorded as time stamps.

We imaged isolated Cy3 molecules and Cy 3 molecule pairs. Each $\mathrm{Cy} 3$ molecule is attached to the $5^{\prime}$ end of a
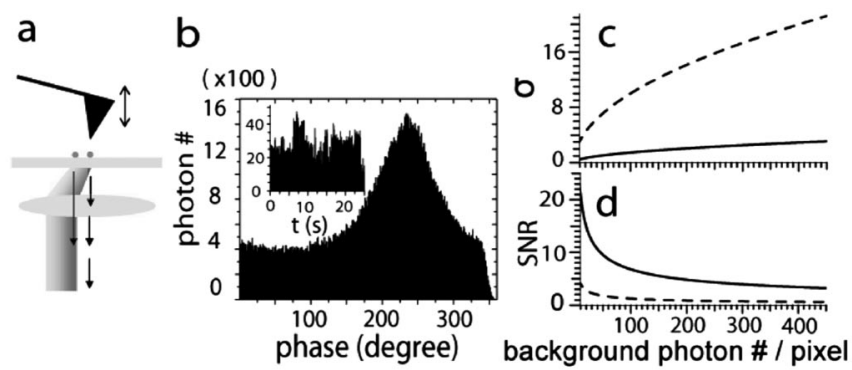

FIG. 1. (a) Illustration of the microscope. The linearly polarized beam, passing through a mask with a wedged window (not shown), is at total internal reflection at the substrate-air interface (focus area $\sim 350 \times 1000 \mathrm{~nm}$ ) to achieve a large field component along the tip axis. (b) Tip-oscillation phase histogram of the photons. The inset is a typical fluorescence time trace of a Cy3 molecule, where the vertical axis is the photon count per $0.01 \mathrm{~s}$. (c) The background noise (standard deviation) obtained from the phase filter (solid curve) and from the unfiltered shot noise, $\sqrt{n}$ (dash curve). The horizontal axis is the same as (d). (d) The SNR calculated as the image pixel signal divided by the background noise from the phase filter (solid curve) and from the unfiltered shot noise (dash curve). The image pixel signal is $0.60 f N / 3(f$ : fluorescence enhancement; $N$ : photon number per pixel emitted by a typical molecule) for the solid curve according to Eq. (1), and $0.75 f N / 3$, which is the direct sum of the near-field photons [Fig. 1(b)], for the dash curve. For both curves, we used $f=5$, $N=10$. 
60-mer single-stranded DNA (ssDNA). Each pair of Cy3 molecules is linked by a $60 \mathrm{bp}$ double-stranded DNA (dsDNA), prepared by annealing two complementary $5^{\prime}$-labeled ssDNA. The dsDNA chain is shorter than the DNA persistence length ( $\sim 150 \mathrm{bp})$, so it is rigid. To obtain topographical atomic force microscope (AFM) images of the DNA molecules, we used glass-mica hybrid slides [24]. To prepare the samples, $1 \mu 1$ of $10 \mathrm{nM}$ DNA solution was spread on the mica surface and evaporated dry, then the surface was rinsed with deionized water and dried with nitrogen gas. The majority of the molecules on the surface produced more than $10^{5}$ photon counts, allowing $>20 \mathrm{~s}$ imaging time.

The signal of fluorescence ANSOM contains the nearfield and far-field components excited by the optical nearfield and propagating laser illumination, respectively. With single molecules, fluorescence enhancement is only $\sim$ twofold to fivefold [Fig. 1(b)], comparable to the fluctuation of the far-field signal [inset of Fig. 1(b)]. In previous work [6], we demonstrated that signal demodulation separates the far-field and near-field signals successfully. This method, however, requires a large number of photons and works only for intense targets, such as quantum dots (Fig. 4 of Ref. [6]). To find an efficient separation method, we studied the $2 \times 10^{5}$ fluorescence photons from an isolated $\mathrm{Cy} 3$ molecule probed by an oscillating tip. Figure 1(b) is the tip-oscillation phase histogram of the photons, from which phase $\phi_{0}$ for the maximum fluorescence enhancement can be determined. It was found from experiments that $\phi_{0}$ remains the same with the same type of tip, and the profile of fluorescence enhancement can be approximated by

$g\left[\phi-\phi_{0}\right]=\exp \left(-\frac{\left|\phi-\phi_{0}\right|_{p}^{2}}{60^{2}}\right) \quad\left(0 \leq \phi, \phi_{0}<360\right)$

where $\left|\phi-\phi_{0}\right|_{p}$ is defined as $\min \left(\left|\phi-\phi_{0}\right|\right.$, $\left.|360-| \phi-\phi_{0}||\right)$. We calculated the raw near-field signal $S_{\text {rn }}$ of a pixel as

$$
S_{r n}=\sum_{\left|\phi-\phi_{0}\right|_{p} \leq 60} \exp \left(-\frac{\left|\phi-\phi_{0}\right|_{p}^{2}}{60^{2}}\right) D[\phi],
$$

where $D[\phi]$ is the number of photons at phase $\phi$. This formula is a bandpass phase filter that passes photons within $60 \mathrm{deg}$ of $\phi_{0}$ [Fig. 1(b)] with weights determined from $g$. The width of the bandpass window was optimized to increase the passed photons and to reduce the bleedthrough between the near-field and far-field signals. The far-field signal $S_{f}$ was calculated as

$$
S_{f}=\sum_{\left|\phi-\left(\phi_{0}-180\right)\right|_{p} \leq 60} \exp \left(-\frac{\left|\phi-\left(\phi_{0}-180\right)\right|_{p}^{2}}{60^{2}}\right) D[\phi]
$$

such that molecules outside the near-field volume of the tip contribute equally to $S_{\mathrm{rn}}$ and $S_{f}$. $S_{f}$ was then averaged with those of its four neighboring pixels to get $\bar{S}_{f}$. The pixel signal was calculated as $S_{n}=S_{\mathrm{rn}}-\bar{S}_{f}$ for $S_{\mathrm{rn}}>\bar{S}_{f}$ and $S_{n}=0$ for $S_{\text {rn }}<\bar{S}_{f}$.

The phase filter effectively suppresses the noise of the background, where we refer to the background as an area without near-field images. One can estimate the effect of the filter by approximating $g$ with a top hat function, with which we can calculate the mean and variance of the background as $\tilde{x}=\sum_{i=0}^{\infty} \sum_{j=0}^{\infty} j P(5 i, 5 n / 3) \times$ $P(i+j, n / 3) \quad$ and $\quad \tilde{\sigma}^{2}=\sum_{i=0}^{\infty} P(5 i, 5 n / 3)\left\{\sum_{j=0}^{\infty}(j-\right.$ $\left.\tilde{x})^{2} P(i+j, n / 3)+\sum_{j=0}^{i} \tilde{x}^{2} P(j, n / 3)\right\}$, respectively, where $i$ and $j$ are dummy variables, $n$ is the average photon number per pixel in the background, and $P(a, b)=$ $\left(e^{-b} b^{a}\right) / a$ ! is the Poisson probability density. The effect of using a Gaussian for $g$ causes only a small change in the standard deviation of the background, giving $\sigma=0.75 \tilde{\sigma}$. Compared with the unfiltered shot noise, the background noise is effectively suppressed with the phase filter [Fig. 1(c)], which provides sixfold improvement in the signal-to-noise ratio (SNR) and makes it possible to image multiple fluorescent targets in the focal spot [Fig. 1(d)]. Assuming that a far-field illuminated molecule emits 10 photons per pixel, good SNR $(>7)$ can be obtained with up to ten molecules in the focal spot and fair SNR $(>3)$ with several tens of molecules in the focal spot [Fig. 1(d)]. The SNR obtained in experiments (Figs. 2 and 4), determined as the difference between the peak image signal and the background baseline divided by the variation of a $100 \times$ $100 \mathrm{~nm}$ background area, is in good agreement with the calculation [Fig. 1(d)].

We imaged 211 isolated single Cy3 molecules. The images are either symmetric [Fig. 2(a)] or elongated [Fig. 2(b)], due to different molecular dipole orientations
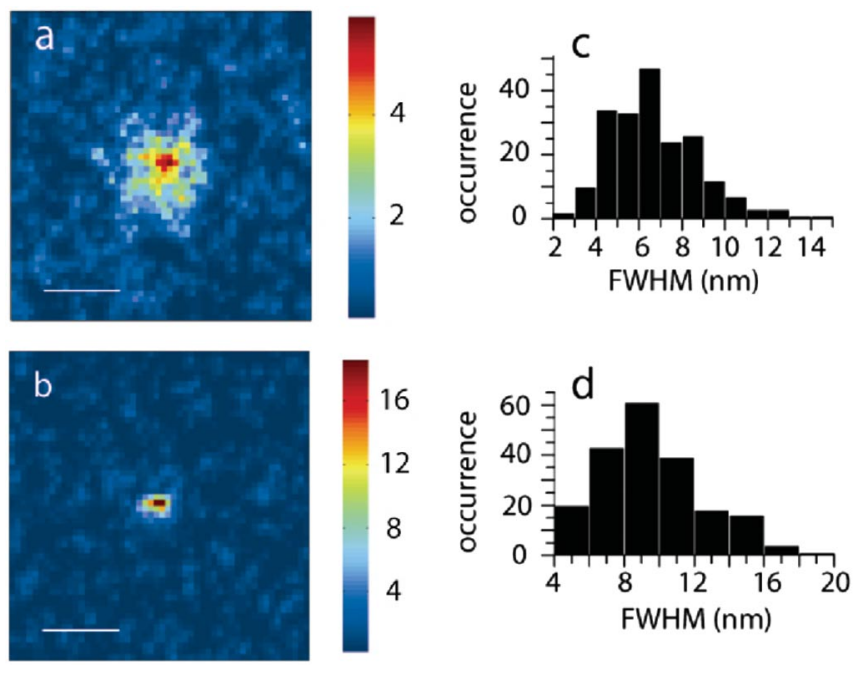

FIG. 2 (color). (a), (b) Near-field images of isolated Cy3 molecules. Each figure was extracted from a $1 \times 1 \mu \mathrm{m}, 512 \times$ 512 pixel image. The SNR for (a) and (b) is 16.2 and 25.5, respectively. Scale bars: $25 \mathrm{~nm}$. (c), (d) Histograms of FWHM measured along the minor and major directions, respectively. 
(discussed below). Histograms of full width at half maximum (FWHM) measured along the minor and major directions of the 211 images are shown in Figs. 2(c) and 2(d), with the average at 6.8 and $9.6 \mathrm{~nm}$, respectively. It is clear that simply choosing the linewidth of a single image is not an accurate method for determining resolution, for the images are highly variable. Here we define an average resolution of $8.2 \mathrm{~nm}$, which is $\sim 3-4$ times better than the previous best ANSOM measurements and close to 1 order better than typical results of apertured near-field microscopy.

To better understand the results, we simulated images of single molecules using the electrostatic dipole model of the tip. This model was adopted in both fluorescence ANSOM and scattering ANSOM [1,25] and was supported by numerical simulations [26]. According to the model, the total field amplitude is

$$
\vec{E}(\vec{r})=E_{0}\left[\hat{z}+\alpha\left(\frac{3 z}{r^{4}} \hat{r}-\frac{1}{r^{3}} \hat{z}\right)\right],
$$

where $E_{0}$ is the external laser field, $\alpha$ is determined experimentally and the coordinate origin is at the tip center. Using $[E(r) \cdot p(\theta, \varphi)]^{2}$ as the image intensity, simulation results [Fig. 3(a)] show that as $\vec{p}$ tilts away from the tip axis, the image becomes elongated and the image center shifts away from the molecule; when $\vec{p}$ is perpendicular to the tip axis $\left(\left|\theta-90^{\circ}\right| \leq 0.8^{\circ}\right)$, the image has two symmetric "lobes"; when $\theta$ is close to $90^{\circ}\left(0.8^{\circ} \leq\right.$ $\left.\left|\theta-90^{\circ}\right| \leq 20^{\circ}\right)$, there is a small region where the signal is below the background. For nonzero $\varphi$, the images are simply $\varphi$-degree rotation of those for $\varphi=0$ [Fig. 3(a)], for the field is symmetric about the tip axis. Simulation patterns for $\theta$ at or close to $90^{\circ}$ were experimentally observed [Figs. 3(b)-3(d)], providing direct support for the electrostatic dipole model.

Measuring true resolution has long been a challenge in near-field microscopy; one of the strongest tests is to make a "Rayleigh" resolution measurement, in which two proximate point sources are resolved. To our knowledge there

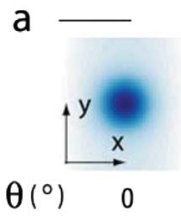

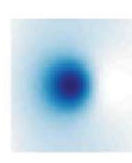

45

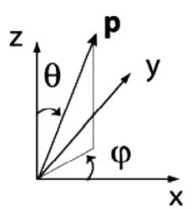

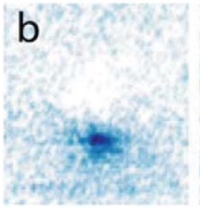

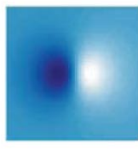

85

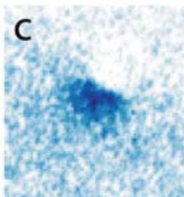

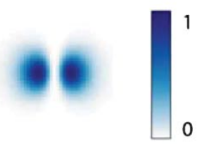

90

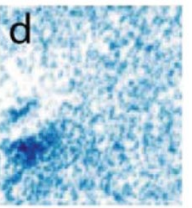

FIG. 3 (color). (a) Simulated images with the tip radius at $10 \mathrm{~nm}$ and fluorescence enhancement at 5 . Scale bar: $20 \mathrm{~nm}$. (b) -(d) Experimental images $(150 \times 150 \mathrm{~nm})$ showing the same patterns as the simulated ones. In these images, $S_{\text {rn }}$ was used as the pixel signal. have been no rigorous near-field measurements such as this made with single molecules, which are excellent approximations of a point source. With the phase filter, we were able to resolve two Cy3 molecules linked by a $60 \mathrm{bp}$ dsDNA oligonucleotide. Figures 4(a)-4(c) are the nearfield optical images of such molecule pairs, where Figs. 4(d)-4(f) are the corresponding topographical AFM images. ANSOM has a better resolution than AFM even with the same tip, because the force involved in AFM, which is proportional to the inverse of the tip-sample distance [27], decays much more slowly than the optical near field. There are no previous AFM experiments that resolved DNA molecules as short as $15 \mathrm{~nm}$; instead, round images for short DNA molecules were observed in this [Fig. 4(d)] and previous experiments [28].

We imaged a total of 389 dsDNA oligonucleotides, 29\% of which showed resolvable $\mathrm{Cy} 3$ pairs. The $\mathrm{Cy} 3$ labeling efficiency for each DNA strand is about $80 \%$, so we expect that $67 \%$ of the optically detectable DNA oligonucleotides are actually labeled with two Cy3 molecules. Factors such as imperfect annealing, photobleaching, and worn tips can all contribute to the failure to resolve the rest. As a control experiment, the 211 images of single Cy 3 molecules were analyzed in the same fashion and double-lobed artifacts were found in only $4 \%$ of the images [Fig. 4(h)], which is a
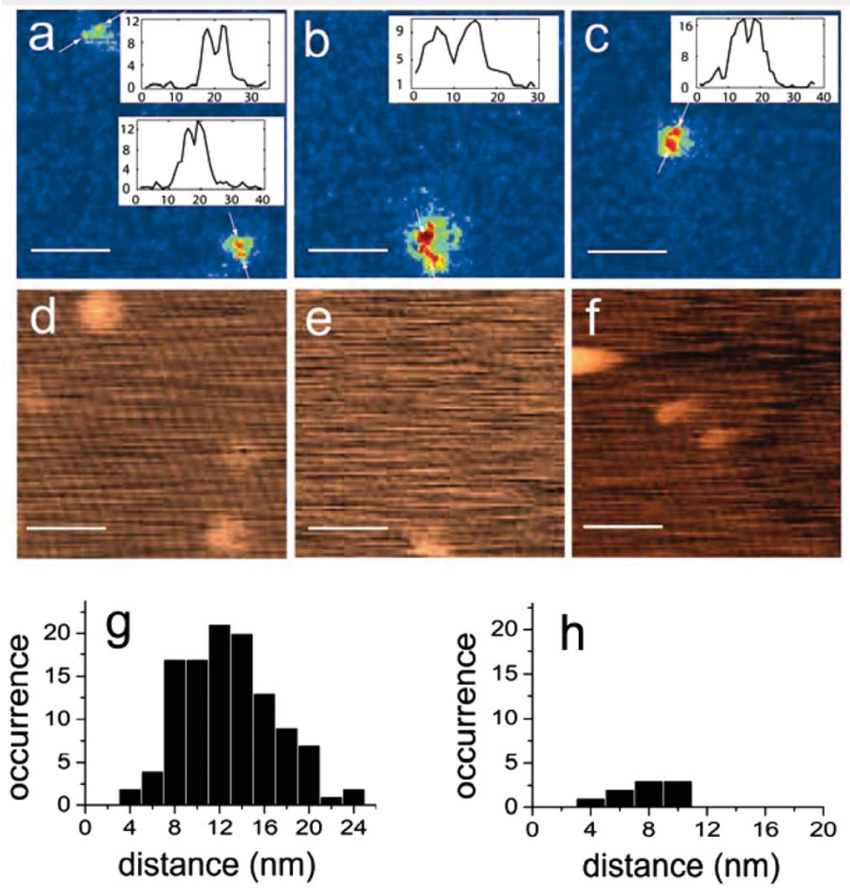

FIG. 4 (color). (a)-(c) Near-field images of Cy3 pairs. The SNR is 12.4 and 15.9 for (a), 16.1 for (b), and 20.4 for (c). The insets show the profiles with line cut through the image centers (indicated by arrows), where the horizontal axis is in pixels ( 1 pixel $=1.95 \mathrm{~nm}$ ) and the vertical axis is the pixel signal. (d)-(f) AFM images corresponding to images (a)-(c), respectively. Scale bars: $50 \mathrm{~nm}$. (g) Histogram of distances between the resolved Cy3 molecules. (h) Histogram of distances between the two artifactual lobes of single Cy3 molecules. 
vast improvement over a previous method which has artifacts in all images [20].

With the resolved $\mathrm{Cy} 3$ molecule pairs, the end-to-end distances of the 60 bp DNA oligonucleotides can be measured [Fig. 4(g)]. The statistical result is $13.0 \mathrm{~nm} \pm$ $4.1 \mathrm{~nm}$ (standard deviation) with standard error of the mean $\sigma_{\mu}=0.4 \mathrm{~nm}$. Random factors that contribute to the distribution of the measurements include limited precision in determining the image centers, shifting of the images, and the flexible carbon linker $(\sim 0.6 \mathrm{~nm})$ between the Cy3 molecule and DNA. Systematic errors also exist in the result. Simulation shows that the two images shift toward each other because the tip enhances both molecules when it is in between them; the shift increases with larger tip radius. The linewidth of the majority of the images indicates an upbound of the tip radius at $15 \mathrm{~nm}$, at which a $2.5 \mathrm{~nm}$ shift was simulated for two molecules separated by $15 \mathrm{~nm}$. Therefore, the precision of our measurement of the DNA length has a statistical error of $3 \%$ and a systematic error up to $20 \%$.

DNA structure depends strongly on humidity and takes the $A$-form and $B$-form structure at low and high humidity, respectively. It is now understood that DNA-binding drugs and proteins can induce local conformational conversion between the two forms [29]. In our experiments, the DNA molecules were imaged at humidity $(\sim 30 \%)$ well below the $73 \%$ threshold for the $A$-form DNA. An unresolved paradox in x-ray diffraction studies of $A$-form DNA is that fibers of long DNA molecules with mixed sequences yield a consistent value of $2.6 \AA / \mathrm{bp}$ for the helical rise [30], but crystal structures of small oligonucleotides $(\sim 10 \mathrm{bp})$ reveal an average value of $2.83 \AA / \mathrm{bp}$ with a standard deviation of $\sim 0.36 \AA / \mathrm{bp}$ across different sequences [31]. The source of the discrepancy is as yet unresolved, although crystal artifacts, molecular weight effects, and incomplete sequence sampling may all play a role. Our measurements described above allow an independent determination of the helical rise, and do not suffer from artifacts due to crystal packing or small molecular weights. The result $(2.17 \AA / b p)$ agrees with the $\mathrm{x}$-ray data of fibers within one sigma of our largest estimated experimental error and falls within the two sigma limit of the sequence-dependent variation observed in crystal structure data.

The phase filtering method should be applicable to nanoantennas [21-23] and supersharp carbon nanotube probes [32] with which both the resolution and the precision can be improved. With the advances of AFM technology, such as imaging in water and fast frame imaging speeds, it may ultimately be possible to combine optical resolution approaching that of electron microscopy with the ability to image biomolecules in physiological conditions.

The authors thank G. Lessard for contributions to the development of initial data acquisition and data analysis algorithms. This work was supported by Pharmagenomix
Inc. and the NIH Director's Pioneer program.

*Present Address: Dept. of Bioengineering, Stanford University and Howard Hughes Medical Institute, Stanford, CA 94305, USA.

${ }^{\dagger}$ Present Address: University of Utah, Dept. of Physics, 115 South 1400 East, Salt Lake City, UT 84112.

[1] F. Zenhausern, Y. Martin, and H. K. Wickramasinghe, Science 269, 1083 (1995).

[2] R. Hillenbrand and F. Keilmann, Appl. Phys. Lett. 80, 25 (2002).

[3] A. Hartschuh et al., Phys. Rev. Lett. 90, 095503 (2003).

[4] T. Ichimura et al., Phys. Rev. Lett. 92, 220801 (2004).

[5] E. J. Sánchez, L. Novotny, and X. S. Xie, Phys. Rev. Lett. 82, 4014 (1999).

[6] J. M. Gerton et al., Phys. Rev. Lett. 93, 180801 (2004).

[7] J. Azoulay et al., Europhys. Lett. 51, 374 (2000).

[8] H. F. Hamann, A. Gallagher, and D. J. Nesbitt, Appl. Phys. Lett. 76, 1953 (2000); H. F. Hamann et al., J. Chem. Phys. 114, 8596 (2001).

[9] V. V. Protasenko et al., Opt. Commun. 210, 11 (2002).

[10] V. V. Protasenko, A. Gallagher, and D. J. Nesbitt, Opt. Commun. 233, 45 (2004).

[11] V. V. Protasenko and A. C. Gallagher, Nano Lett. 4, 1329 (2004).

[12] J. Azoulay et al., J. Microsc. 194, 486 (1999).

[13] N. Hayazawa, I. Inouye, and S. Kawata, J. Microsc. 194, 472 (1999).

[14] A. Kramer et al., Appl. Phys. Lett. 80, 1652 (2002).

[15] T. J. Yang, G. A. Lessard, and S. R. Quake, Appl. Phys. Lett. 76, 378 (2000).

[16] W. Trabesinger et al., J. Microsc. 209, 249 (2003); W. Trabesinger et al., Appl. Phys. Lett. 81, 2118 (2002).

[17] S. Weiss, Science 283, 1676 (1999).

[18] W. E. Moerner and M. Orrit, Science 283, 1670 (1999).

[19] E. Betzig and R. J. Chichester, Science 262, 1422 (1993).

[20] H. G. Frey et al., Phys. Rev. Lett. 93, 200801 (2004).

[21] P. J. Schuck et al., Phys. Rev. Lett. 94, 017402 (2005).

[22] P. Mühlschlegel et al., Science 308, 1607 (2005).

[23] J. N. Farahani et al., Phys. Rev. Lett. 95, 017402 (2005).

[24] A thin layer $(\sim 2 \mu \mathrm{m})$ of mica was lifted off a mica sheet and placed on a clean glass cover slide; bonding between the two surfaces is immediate, probably due to van der Waals forces. The mica surface was treated with $20 \mathrm{mM} \mathrm{MgCl}$ and rinsed by deionized water.

[25] B. Knolland and F. Keilmann, Nature (London) 399, 134 (1999).

[26] A. Bouhelier et al., Phys. Rev. Lett. 90, 013903 (2003).

[27] F. J. Giessibl, Rev. Mod. Phys. 75, 949 (2003).

[28] H. G. Hansma, Nucleic Acids Res. 24, 713 (1996).

[29] X.-J. Lu, Z. Shakked, and W. K. Olson, J. Mol. Biol. 300, 819 (2000).

[30] S. B. Zimmerman, Annu. Rev. Biochem. 51, 395 (1982).

[31] X.-J. Lu and W. K. Olson, Nucleic Acids Res. 31, 5108 (2003).

[32] J. H. Hafner et al., J. Phys. Chem. B 105, 743 (2001); L. A. Wade et al., Nano Lett. 4, 725 (2004). 